\title{
Variational Iteration Method for the Solution of Differential Equation of Motion of the Mathematical Pendulum and Duffing-Harmonic Oscillator
}

\section{Muhammad Munib Khan}

Department of Basic Sciences, University of Engineering and Technology Peshawar, Pakistan

\begin{abstract}
In this work, the differential equation of motion of the undamped mathematical pendulum and Duffing-harmonic oscillator are discussed by using the variational iteration method. Additionally, common problems of pendulum are classified and Lagrange multipliers are obtained for each type of problem. Examples are given for illustration.
\end{abstract}

\section{Introduction}

Vibration of dynamical systems can be divided into two main classes like discrete and distributed. The variables in discrete systems depend on time only, whereas in distributed systems such as beams, plates, etc. variables depend on time and space. Therefore, equations of motion of discrete systems are described by ordinary differential equations, while equations of motion of distributed systems are described by partial differential equations [1].

The variational iteration method [2-13] has been used to solve many nonlinear PDEs, ordinary differential equations such that wave solutions, rational solutions, compacton solutions and other types of solution were found by Abdou and Soliman [14]. Additionally, He [15] used VIM to solve linear/nonlinear vibration problems.

Received: April 16, 2019; Revised: April 23, 2019; Accepted: April 24, 2019

2010 Mathematics Subject Classification: 34-XX.

Keywords and phrases: variational iteration method, vibration, Lagrange multiplier.

Copyright (C) 2019 Muhammad Munib Khan. This is an open access article distributed under the Creative Commons Attribution License, which permits unrestricted use, distribution, and reproduction in any medium, provided the original work is properly cited. 
The procedure presented in this paper can be simply extended to solve more complex vibration problems; such as aeroelasticity, random vibrations etc.

\section{Variational Iteration Method}

In order to illustrate the basic concepts of VIM, the following nonlinear partial differential equation can be considered

$$
R u(x, t)+N u(x, t)=g(x, t),
$$

where $R$ is a linear operator which has partial derivatives with respect to $N u(x, t)$ is a nonlinear term and $g(x, t)$ is an inhomogeneous term.

According to VIM, the following iteration formula can be constructed.

$$
u_{n+1}(x, t)=u_{n}(x, t)+\int_{0}^{t} \lambda\left[R \tilde{u}_{n}+N \tilde{u}_{n}-g\right] d \tau,
$$

where $\lambda$ is the general Lagrange multiplier which can be identified optimally via variational theory, $R \tilde{u}_{n}$ and $N \tilde{u}_{n}$ are considered as restricted variations, i.e.,

$$
\delta R \tilde{u}_{n}=0, \quad \delta N \tilde{u}_{n}=0 .
$$

\section{Examples}

\section{Example 1.}

In this example, Mathematical Pendulum that was studied by $\mathrm{He}[15,16]$ is considered.

The differential equation of motion of the undamped mathematical pendulum is given by

$$
\ddot{y}+\omega^{2} \sin y=0 .
$$

The initial conditions for this problem are as follows:

$$
\begin{aligned}
& y(0)=A, \\
& \dot{y}(0)=0 .
\end{aligned}
$$


The sin $y$ term in Eq. (3) is a nonlinear term and it can be expanded as

$$
\sin y \approx y-\frac{1}{6} y^{3}
$$

Substituting Eq. (4) into Eq. (5) gives

$$
\ddot{y}+\omega^{2} y-\frac{\omega^{2}}{6} y^{3}=0 .
$$

A more detailed form of this mathematical pendulum was investigated by $\mathrm{He}[15$, $16]$.

The Lagrange multiplier of this problem is

$$
\lambda=\frac{1}{\omega} \sin [\omega(\tau-t)]
$$

Hence the iteration formula is

$$
y_{n+1}(t)=y_{n}(t)+\frac{1}{\omega} \int_{0}^{t} \sin [\omega(\tau-t)]\left[y^{\prime \prime}(\tau)+\omega^{2} y(\tau)-\frac{\omega^{2}}{6} y^{3}(\tau)\right] d \tau .
$$

The complementary solution of this problem that is used as an initial approximation is given by

$$
y_{0}(t)=A \cos (\alpha \omega t)
$$

where $\alpha$ is an unknown constant.

Substituting the initial approximation into Eq. (5), the following residual is obtained

$$
\begin{aligned}
R_{0}(t) & \approx \ddot{y}+\omega^{2} y-\frac{\omega^{2}}{6} y^{3} \\
& =A\left(1-\frac{1}{8} A^{2}-\alpha^{2}\right) \omega^{2} \cos (\alpha \omega t)-\frac{1}{24} A^{3} \omega^{2} \cos (3 \alpha \omega t) .
\end{aligned}
$$

The coefficient of the $\cos (\alpha \omega t)$ term is set to zero in order to eliminate the secular term which may occur in the next iteration. Doing so, the expression of $\alpha$ is found as follows 


$$
\alpha=\sqrt{1-\frac{A^{2}}{8}}
$$

Hence,

$$
y_{1}(t)=A \cos \alpha \omega t-\frac{A^{3}}{24\left(9 \alpha^{2}-1\right) \omega^{2}}(\cos 3 \alpha \omega t-\cos \omega t)
$$

with $\alpha$ defined in Eq. (11).

The period can be expressed as follows

$$
T=\frac{2 \pi}{\omega \sqrt{1-\frac{1}{8} A^{2}}} .
$$

If $A=\frac{\pi}{2}$, then $T=1.20 T_{0}$. On the other hand He's $[15,16]$ approximation gives $T=1.17 T_{0}$, while the exact period is $T_{e x}=1.16 T_{0}$, where $T_{0}=2 \pi / \omega$.

\section{Example 2.}

In this example, the problem that was studied by Nayfeh and Mook [17] is considered.

The differential equation of motion is given by,

$$
\ddot{u}+\omega^{2} u+\varepsilon u^{2} \ddot{u}=0 .
$$

The initial conditions for this problem are as follows:

$$
\begin{gathered}
y(0)=A, \\
\dot{y}(0)=0 .
\end{gathered}
$$

The Lagrange multiplier of this problem is

$$
\lambda=\frac{1}{\omega} \sin [\omega(\tau-t)]
$$

The iteration formula is given by

$$
u_{n+1}(t)=u_{n}(t)+\frac{1}{\omega} \int_{0}^{t} \sin [\omega(\tau-t)]\left[u^{\prime \prime}(\tau)+\omega^{2} u(\tau)+\varepsilon u^{2}(\tau) u^{\prime \prime}(\tau)\right] d \tau
$$


The complementary solution of this problem that is used as an initial approximation is given by

$$
u_{0}(t)=A \cos (\alpha \omega t)
$$

where $\alpha$ is an unknown constant.

Substituting the initial approximation given by Eq. (18), the following residual is obtained as follows

$$
\begin{aligned}
R_{0}(t) & \approx \ddot{u}+\omega^{2} u+\varepsilon u^{2} \ddot{u} \\
& =A \omega^{2}\left(1-\alpha^{2}-\frac{3}{4} A^{2} \varepsilon\right) \cos (\alpha \omega t)-\frac{1}{4} \varepsilon A^{3} \alpha^{2} \omega^{2} \cos (3 \alpha \omega t) .
\end{aligned}
$$

The coefficient of the $\cos (\alpha \omega t)$ term is set to zero in order to eliminate the secular term which may occur in the next iteration. Doing so, the expression for $\alpha$ is obtained as follows

$$
\alpha=\frac{2}{\sqrt{4+3 \varepsilon A^{2}}}
$$

Hence,

$$
y_{1}(t)=A \cos \alpha \omega t+\frac{\varepsilon A^{3} \alpha^{2}}{4\left(9 \alpha^{2}-1\right)}(\cos \omega t-\cos 3 \alpha \omega t)
$$

with $\alpha$ defined in Eq. (20).

The new frequency is defined as follows

$$
\omega_{1}=\alpha \omega \Rightarrow \omega_{1}=\frac{2}{\sqrt{4+3 \varepsilon A^{2}}} \omega .
$$

The frequency that is obtained by Nayfeh and Mook [17] using the perturbation method is

$$
\omega_{1}=\omega\left(1-\frac{3}{8} \varepsilon A^{2}\right)
$$

Note that Eq. (23) is valid only for small $\varepsilon$ values. However, the frequency expression given by Eq. (22) is valid for all $\varepsilon$ values and takes the following form for small $\varepsilon$ 
values

$$
\omega_{1} \approx 1-\frac{3}{8} \varepsilon A^{2}+\frac{27}{128} \varepsilon^{2} A^{4}+\cdots .
$$

\section{Example 3.}

In this example, the Duffing-harmonic oscillator that was studied by Mickens [18] and Lim and $\mathrm{Wu}[19]$ is considered.

The differential equation of motion is given by,

$$
\frac{d^{2} y}{d t^{2}}+\frac{y^{3}}{1+y^{2}}=0 .
$$

The initial conditions for this problem are as follows:

$$
\begin{aligned}
& y(0)=A, \\
& \dot{y}(0)=0 .
\end{aligned}
$$

For small $y$ values, Eq. (25) reduces to

$$
\frac{d^{2} y}{d t^{2}}+y^{3} \simeq 0
$$

On the other hand, for large $y$ values, Eq. (25) reduces to

$$
\frac{d^{2} y}{d t^{2}}+y \simeq 0 .
$$

Considering Eqs. (26a) and (26b) respectively, it is noticed that for small $y$ values, Eq. (27) reduces to the equation of motion of the Duffing-type nonlinear oscillator while for large $y$ values, it reduces to the equation of motion of a linear harmonic oscillator. Therefore, Eq. (27) is called as Duffing-harmonic oscillator equation of motion.

The following form of Eq. (27) is going to be studied in this example

$$
\left(1+y^{2}\right) \frac{d^{2} y}{d t^{2}}+y^{3}=0 .
$$

He's technique is going to be used to overcome seculer terms that appear in the 
iterations. The initial approximation is,

$$
y_{0}(t)=A \cos (\alpha t)
$$

where $\alpha$ is an unknown constant.

Substituting the initial approximation into Eq. (28), the following residual is obtained

$$
\begin{aligned}
R_{0}(t) & \approx\left(1+y^{2}\right) \ddot{y}+y^{3} \\
& =\left(\frac{3}{4} A^{2}-\alpha^{2}-\frac{3}{4} A^{2} \alpha^{2}\right) \cos (\alpha t)+\frac{A^{3}}{4}\left(1-\alpha^{2}\right) \cos (3 \alpha t) .
\end{aligned}
$$

In order to discard the seculer terms, the coefficient of $\cos (\alpha t)$ is set to zero which gives the expression of $\alpha$ as follows

$$
\alpha=\sqrt{\frac{\frac{3}{4} A^{2}}{1+\frac{3}{4} A^{2}}} .
$$

Hence the new frequency is defined as follows

$$
\omega=\sqrt{\frac{\frac{3}{4} A^{2}}{1+\frac{3}{4} A^{2}}}
$$

which is the same with the one found by Mickens [18].

The iteration formula is given by

$$
u_{1}(t)=u_{0}(t)+\int_{0}^{t}(\tau-1)\left[\frac{A^{3}}{4}\left(1-\alpha^{2}\right) \cos (3 \alpha \tau)\right] d \tau
$$

Hence,

$$
y_{1}(t)=\cos \omega t+\frac{A}{27}(\cos 3 \omega t-1)
$$

with $\omega$ defined in Eq. (30). 
For small values of amplitude $A$, the frequency expression given in Eq. (32) is expressed as follows

$$
\omega \simeq \sqrt{\frac{3}{4}} A
$$

Additionally, for large values of amplitude $A$, the frequency expression given in Eq. (32) is expressed as follows

$$
\omega \simeq 1
$$

which agree with the approximations made for the equations of motion.

\section{Conclusion}

In this work, the differential equation of motion of the undamped mathematical pendulum and Duffing-harmonic oscillator are solved using the variational iteration method. Additionally, the procedure presented in this paper can be simply extended to solve more complex vibration problems; such as aeroelasticity, random vibrations etc.

\section{References}

[1] L. Meirovitch, Computational Methods in Structural Dynamics, Sijthoff \& Noordhoff, The Netherlands: Alphen aan den Rijn, 1980.

[2] J.-H. He, Variational approach to the Thomas-Fermi equation, Appl. Math. Comput. 143(2-3) (2003), 533-535. https://doi.org/10.1016/S0096-3003(02)00380-6

[3] J.-H. He, G.-C. Wu and F. Austin, The variational iteration method which should be followed, Nonlinear Sci. Lett. A 1(1) (2010), 1-30.

[4] J.-H. He and X.-H. Wu, Variational iteration method: New development and applications, Comput. Math. Appl. 54(7-8) (2007), 881-894.

https://doi.org/10.1016/j.camwa.2006.12.083

[5] J.-H. He, Variational iteration method-Some recent results and new interpretations, $J$. Comput. Appl. Math. 207(1) (2007), 3-17. https://doi.org/10.1016/j.cam.2006.07.009

[6] J.-H. He, Some asymptotic methods for strongly nonlinear equations, Int. J. Mod. Phys. B 20(10) (2006), 1141-1199. https://doi.org/10.1142/S0217979206033796

[7] M. Rafiq, H. Ahmad, and S. T. Mohyud-Din, Variational iteration method with an auxiliary parameter for solving Volterra's population model, Nonlinear Sci. Lett. A 8(4) (2017), 389-396. 
[8] M. Nadeem, F. Li, and H. Ahmad, Modified Laplace variational iteration method for solving fourth-order parabolic partial differential equation with variable coefficients, Comput. Math. Appl., 2019. https://doi.org/10.1016/j.camwa.2019.03.053

[9] H. Ahmad, Variational iteration algorithm-I with an auxiliary parameter for solving Fokker-Planck equation, Earthline J. Math. Sci. 2(1) (2019), 29-37.

https://doi.org/10.34198/ejms.2119.2937

[10] H. Ahmad, Variational iteration method with an auxiliary parameter for solving telegraph equations, J. Nonlinear Anal. Appl. 2018(2) (2018), 223-232.

[11] H. Ahmad, Variational iteration algorithm-I with an auxiliary parameter for wave-like vibration equations, J. Low Freq. Noise, Vib. Act. Control, 2019. https://doi.org/10.1177/1461348418823126

[12] H. Ahmad, Auxiliary parameter in the variational iteration algorithm-II and its optimal determination, Nonlinear Sci. Lett. A 9(1) (2018), 62-72.

[13] H. Ahmad, Variational iteration method with an auxiliary parameter for solving differential equations of the fifth order, Nonlinear Sci. Lett. A 9(1) (2018), 27-35.

[14] M. A. Abdou and A. A. Soliman, Variational iteration method for solving Burger's and coupled Burger's equations, J. Comput. Appl. Math. 181(2) (2005), 245-251. https://doi.org/10.1016/j.cam.2004.11.032

[15] J.-H. He, Iteration perturbation method for strongly nonlinear oscillations, J. Vib. Control. 7(5) (2001), 631-642. https://doi.org/10.1177/107754630100700501

[16] D.-N. Yu, J.-H. He and A. G. Garcia, Homotopy perturbation method with an auxiliary parameter for nonlinear oscillators, J. Low Freq. Noise, Vib. Act. Control, 2018. https://doi.org/10.1177/1461348418811028

[17] A. H. Nayfeh and D. T. Mook, Nonlinear Oscillations, Wiley, 1979. https://doi.org/10.1115/1.3153771

[18] R. E. Mickens, Mathematical and numerical study of the Duffing-harmonic oscillator, Journal of Sound and Vibration 244(3) (2001), 563-567.

https://doi.org/10.1006/jsvi.2000.3502

[19] C.W. Lim and B. S. Wu, A new analytical approach to the Duffing-harmonic oscillator, Phys. Lett. A 311 (2003), 365-373. https://doi.org/10.1016/S0375-9601(03)00513-9 